\title{
Mineralogical controls on the retention and chemical composition of dissolved pyrogenic carbon
}

FERnANDA SANTOS ${ }^{1 *}$, Jing YAN ${ }^{2}$, HUi LI ${ }^{3}$, Elizabeth M. HERNDON $^{4}$, Sanjai ParikH ${ }^{5}$, Teamrat GHEZZEhei ${ }^{6}$, FranCoIS Blanchette $^{7}$, JEFFrey A. Bird ${ }^{8}$, ASMEREt Asefaw Berhe ${ }^{9}$

${ }^{1}$ Life and Environmental Sciences, University of CaliforniaMerced, Merced, CA and Environmental Sciences Division, Oak Ridge National Laboratory, Oak Ridge, TN, [*correspondence: santosf@ornl.gov]

${ }^{2}$ Life and Environmental Sciences, University of CaliforniaMerced, Merced, CA, [jyan235@ucmerced.edu]

${ }^{3}$ Environmental Sciences Division, Oak Ridge National Laboratory, Oak Ridge, TN, [lih4@ornl.gov]

${ }^{4}$ Environmental Sciences Division, Oak Ridge National Laboratory, Oak Ridge, TN, [herndonem@ornl.gov] ${ }^{5}$ Department of Land, Air and Water Resources, University of California, Davis, Davis, CA, [sjparikh@ucdavis.edu]

${ }^{6}$ Life and Environmental Sciences, University of CaliforniaMerced, Merced, CA, [taghezzehei@ucmerced.edu]

${ }^{7}$ Applied Mathematics, University of California, Merced, Merced, CA, [fblanchette@ucmerced.edu]

${ }^{8}$ School of Earth and Environmental Sciences, Queens College, City University of New York, Flushing, NY, [Jeffrey.Bird@qc.cuny.edu]

${ }^{9}$ Life and Environmental Sciences, University of CaliforniaMerced, Merced, CA, [aaberhe@ucmerced.edu]

Increased wildfire events raise the need to understand the factors driving the watershed export of organic contaminants co-occurring with fire-transformed carbon-rich residues known as pyrogenic carbon (PyC). The impacts of soil minerals on PyC transport through the soil-aquatic continuum, however, remain unclear. We conducted soil adsorption experiments to examine the influence of quartz, kaolinite, hematite, and g-manganese oxide on the: retention behavior of dissolved $(<0.45 \mu \mathrm{m}$ pore size) PyC (DPyC) over time, and chemical transformation of retained and mobilized DPyC. Sorption increased from silica to kaolinite to hematite, and with increasing DPyC concentration at or close to the equilibrium, suggesting that mineral composition regulates DPyC transport. Sorption rates became more stable after 3-5 hours of contact time, indicating that DPyC sorption onto mineral surfaces is a relatively fast process. Additionally, sorption rate constants decreased with increasing DPyC concentration for most of the cases, suggesting that sorption is less affected by mineral type, but largely dependent on mineral sorption capacity. Liquid-state ${ }^{1} \mathrm{H}-\mathrm{NMR}$ spectroscopy of DPyC in solution indicated that the presence of hematite preferentially retained simple lipids while kaolinite preferentially retained functionalized aliphatics Finally, we will show how manganese oxides affected the retention and composition of DPyC. 